\title{
Vino nuevo en odres viejos ${ }^{1}$
}

\section{David Anisi}

'Nadie pone remiendo de paño nuevo en vestido viejo; porque tal remiendo tira del vestido, y se hace peor la rotura.

$\mathrm{Ni}$ echan vino nuevo en odres viejos; de otra manera los odres se rompen, y el vino se desparrama, y los odres se pierden; pero echan el vino nuevo en odres nuevos, y lo uno y lo otro se conservan conjuntamente".

(Mateo 9; 16,17)

El primer número de 1991 de The Economic Journal, por aquello de cumplirse el segundo centenario de la publicación, se dedicó a recoger las respuestas que distinguidos economistas habían dado a la pregunta " $\mathrm{Y}$ los próximos cien años?".

Allí estaban las opiniones de Baumol, Bhagwati, Buchanan, Dasgupta, Fishburn, Friedman, Galbraith, Hahn, Johnston, Kay, Malinvaud, Morishima, Oswald, Pencavel, Plott, Robinson, Roth, Schmalensee, Stern, Stiglitz, Turnovsky y Wiseman ${ }^{2}$.

Frente a tales Gigantes poco puede significar la opinión de un David ${ }^{3}$. Pero

1. Agradezco sus comentarios a V. Donoso, F. Esteve y R. Muñoz de Bustillo.

2. The Economic Journal. Vol. 101, n²404, January 1991.

3. Trato de rendir con esto, un homenaje a D.H. Robertson quien, precisamente también utilizando las páginas de The Economic Journal, y haciendo referencia a la polémica sobre "las cajas vacías" entre Pigou y Clapham comenzaba su artículo de 1924 con estas palabras:

"Hace un año las páginas de esta revista se vieron animadas por una batalla de gigantes (...). Mi propósito es otro más presuntuoso: desafiar -aunque provisto de un arma tan rudimentaria como la de David- a uno de los gigantes en su propio terreno, y lanzar una china que, aunque parezca inofensiva vista desde su augusta frente, quizá pueda provocar al menos un rugido de explicación".

The Economic Journal, vol. XXXIV (1924), pág. 16.

Reproducido en Stigler, G.L. y Boulding K.E. (1957): Ensayos sobre la Teoría de los Precios. Aguilar, Madrid, 1968. 
todavía existo y puedo hablar. Además, fueron precisamente esos, y otros más, mis maestros; aquellos que, queriéndolo o no, me enseñaron a manejar la honda y a elegir cuidadosamente las piedras.

Milton Friedman titula su contribución: "Old Wine in New Bottles"4. Yo, desde la distancia que me impone la estatura de tal Goliath, y desde un respeto imponente, me atrevo a entrar en liza alterando, en el título, subversivamente el lema.

\section{TEORÍA ECONÓMICA y OTRas Cosas}

La verdad es que me sorprendo por lo populares que somos. Cualquier periódico de difusión mundial o local incluye una sección de "Economía". Y nos parece normal. Aunque alguien podría tal vez preguntarse por qué no aparecen en esos mismos periódicos secciones diarias bajo los grandes títulos de "Optica", o "Física Cuántica", o "Matemáticas", o "Geografía", o...

Claro que siempre se podría argumentar que tales otrạs materias sólo afectan a unos pocos, y que, en consecuencia, esos individuos acudirán a sus revistas especializadas donde podrán informarse de los eventos que les emocionen. Pero también es cierto que el dolor, la enfermedad y el saludo de la muerte nos afecta a todos, y no por ello se incluye en esos papeles cotidianos una sección que se titule "Medicina".

Poco se de Medicina, aunque algo leo y de algo me informan. Pero conozco lo suficiente para saber que si, en el caso de una hipotética Sección de Medicina en un hipotético diario, se leyera, por ejemplo: "El Ministro de Sanidad afirma que el ajo es el mejor remedio para el cáncer de mama", las carcajadas de los médicos y la repulsa generalizada ante tal desvergüenza harían que precisamente resultara hipotética tal Sección e hipotético el periódico donde se publicara.

Y no es que, de por sí, la hipótesis "el ajo es el mejor remedio para el cáncer de mama" sea desechable. Simplemente la Profesión Médica preguntaría al dicente sobre el origen de su ocurrencia. Trataría de establecer, dentro de la Historia de la Medicina, cuando, en todo caso, tal hipótesis, o otras iguales o similares, fueron formuladas, cómo se validaron o falsaron, bajo qué criterios endocrinológicos podría realizarse tal afirmación, cuáles fueron o son los resultados de la contrastación empírica, donde están los conocimientos que fundamentan tal hipótesis, etc. etc.

4. Friedman, M. (1991): "Old Wine in New Bottles". The Economic Journal, Vol. 101, $\mathrm{n}^{2} 404$, January 1991 , pp. 33-40. 
Pero si en nuestros periódicos, no hipotéticos sino reales, y en nuestra real -y no hipotética- sección de Economía, el señor Ministro de la Cosa dice: "Ha sido la elevación de los salarios monetarios la responsable del aumento del desempleo", sólo muy pocos, y con muy poco poder se preguntan o ríen diciéndose: ¿Bajo qué supuestos afirmas tal cosa?, ¿En qué modelo te mueves?, ¿Cuál ha sido y es la validez empírica de tu hipótesis?, ¿En qué lugar de la historia del Análisis Económico te has quedado?

Y recalco "con muy poco poder" porque está claro que esos periódicos y esas secciones son reales, cotidianos. Cualquier persona, incluso un ministro, se siente capacitado para opinar sobre economía amparándose en la más completa impunidad a la hora de decir insensateces.

Y en esa prensa, día tras día, se defiende la opción del mercado como solución a todos los problemas. Si alguno existe será porque el mercado correspondiente no funciona. $\mathrm{Y}$ así sucesivamente.

Trasmiten cosas tales como que todas las convulsiones sociales de la antigua -y tal vez hoy renovada- URSS y de la conocida como "Europa del Este" se arreglarían con más mercado. Dicen, y todos lo leemos sin sonrojarnos, que Fulanito y Menganito han afirmado que el mercado y la privatización es el remedio para los males de América Latina, para la Sanidad Pública, para...

Y entretanto, nosotros, los teóricos de esa cosa llamada Economía, callados frente a tanta expresión de ignorancia. Derrotados de antemano puesto que nuestra batalla no puede darse sobre una pizarra, en comunicaciones o artículos. Nuestra batalla es contra una propaganda machacona y acientífica que "vende" el mercado como la Coca-Cola.

Pero este mismo fenómeno debe darnos pie para reflexión. ¿Qué se sabe desde la Teoría Económica sobre el mercado? Esa publicidad ¿está respaldada por nuestros conocimientos teóricos? Y, si no lo está, entonces ¿por qué? ¿Quienes son esos economistas que parece apoyan esa idea?

Decía al principio que me sorprendo por lo populares que somos. También, como se habrá visto, me preocupo. Quizá podamos decir que la Medicina es lo que hacen los médicos, la Geología lo que hacen los geólogos, etc... Pero no creo que la Economía, a pesar de lo popular entre nosotros de la expresión, sea lo que hacen los economistas ${ }^{5}$.

5. Todos atribuimos esa expresión a Viner, pero no he logrado encontrar la referencia apropiada. Parece que a Hansen (1991), por lo que relata en su nota 1 le ocurre lo mismo. Hansen, W.L. (1991): "The Education of The Executive Secretary of The American Economic Association's Commission on Graduate Education in Economics". Journal of Economic Literature. Vol. XXIX, Septembre 1991. pp. 1054-1087. 
Y esto por varias razones. En primer lugar porque si al hablar de "Economía" entendemos por tal "Actividad Económica", está claro que ésta se lleva a cabo por los denominados "agentes económicos"; y los economistas participan en esa "actividad económica" de forma análoga, y con el mismo status que los médicos, filósofos, reyes o ladrones: esto es, con sus decisiones, más o menos importantes, más o menos trascendentes. Y con su participación, más o menos relevante, más o menos significativa, en las formas en las que cada sociedad resuelve el problema económico.

En segundo lugar, porque tampoco está tan claro qué es lo que hacen los economistas como profesión. En principio, en nuestro hoy y ahora occidental y desarrollado, un economista es aquel individuo que así se define, es decir aquella persona sobre la que algún Estado o Universidad certifica que cursó los estudios correspondientes. Y esos estudios, como todos sabemos, contienen materias de lo más diversas.

Sea como sea, y en sus variados puestos de trabajo, pocos serían aquellos "economistas" que dirían de sí mismos que usan su tiempo para tratar de estudiar científicamente la forma en que una determinada sociedad resuelve el problema económico 6 .

Decir que alguno de nosotros hacemos "Ciencia Económica", mientras que otros practicamos el "Arte Económico", tampoco sirve de mucho puesto que entraríamos con ello de lleno en toda la polémica metodológica sobre la cientificidad de nuestra ocupación, y no se trata de tal cosa en estos momentos.

Por ello, tal vez resulte conveniente retomar la vieja distinción schumpete1966):

6. Dice Friedman (Friedman, M. (1962): Teoría de los Precios. Alianza Editorial, Madrid

"La economía es la ciencia que estudia la forma en que una determinada sociedad resuelve sus problemas económicos. Existe un problema económico cuando se usan medios escasos para satisfacer fines alternativos". (pág. 13).

y posteriormente continúa:

"La ciencia económica, en nuestra definición, no se ocupa de todos los problemas económicos. Es una ciencia social y, por lo tanto, trata principalmente de los problemas económicos cuyas soluciones implican la cooperación e interacción de diferentes individuos. Considera problemas que afectan a una sola persona en cuanto la conducta de esta implica o afecta a otras personas. Además no trata el problema económico en sentido abstracto sino de la manera en que una determinada sociedad resuelve sus problemas económicos." (pág. 14).

(los subrayados son del autor).

7. La distinción entre Ciencia y Arte en lo que se refiere a lo económico se establece en Keynes, J. Neville (1891): The Scope and Method of Political Economy. Macmillan, London, 1891. (Recogido y citado por Friedman, M. (1952): "La Metodología de la Economía Positiva" en Friedman, M. (1953): Ensayos sobre Economia Positiva. Gredos, Madrid, 1967). 
riana entre Análisis Económico y Pensamiento Económico para tratar de aclarar o delimitar los distintos campos ${ }^{8}$.

Por otro lado pienso, con Pigou ${ }^{9}$, que no es mi misión, en cuanto un tipo particular de economista, enseñar a vender más y mejor, en cualquiera de sus acepciones o formas, sino explicar y explicarme precisamente el por qué de esas ventas y los procesos de decisión sobre las que se sustentan.

Trataré, en consecuencia, de distinguir dentro de la profesión aquellas actividades que se realizan compartiendo con el resto de los individuos la categoría de simples agentes económicos, aquellas que de alguna u otra forma se dirigen al fomento de las ventas de las instituciones para las que se trabaja, esas otras más que podrían incluirse dentro del "Pensamiento Económico" y, por fin, las dirigidas e incluidas en el campo del Análisis Económico.

Mucho de simple relato de la actividad económica se encuentra en esas secciones de los periódicos, y mucho también de pura propaganda de aquel que a fin de cuentas paga; e incluso, de vez en cuando, aunque difícilmente distinguible de lo anterior, algo también vemos de Pensamiento Económico. Pero el Análisis Económico, o la Teoría Económica, si se prefiere, se difunde en otros lugares.

\section{PERO, ¿QUÉ ES EL ANÁLISIS ECONÓMICO?}

En principio, el Análisis Económico recogería el conjunto de instrumentos, hipótesis y teorías dirigido a la construcción de una Ciencia que, por continuar con la definición de Friedman ${ }^{10}$, tratara de estudiar cómo una determinada sociedad resuelve el problema económico, entendiendo éste como la existencia de medios escasos para la consecución de fines alternativos.

\section{Dice Schumpeter:}

"Entiendo por historia del Análisis Económico la historia de los esfuerzos intelectuales realizados por los hombres para entender los fenómenos económicos, o bien-redundando en lo mismo- la historia de los aspectos analíticos o científicos del pensamiento económico" (pág. 37).

(El subrayado es del autor).

Schumpeter, J.A. (1954): Historia del Análisis Económico. Ariel, Barcelona, 1971.

9. Dice Pigou a este respecto:

“... no es oficio de los economistas enseñar a los fabricantes de lana a transformar y vender la lana, ni enseñar a los cerveceros a fabricar y vender cerveza, ni a desempeñar su oficio a cualquier otro hombre de negocios". (pág. 127).

Pigou, A.C. (1922): "Las cajas vacías económicas: respuesta", recogido en Stigler, G.J. y Boulding, K.E. (1957). (Ver nota 2).

10. Ver nota 4. 
La propia historia del desarrollo de este Análisis ha determinado la triple dimensión del problema que, plasmado en la forma ya habitual de los manuales introductorios se resume en las ya clásicas tres preguntas: ¿qué?, ¿cómo? y ¿para quién?, y en las también clásicas tres formas de contestarlas: Jerarquía, Mercado, Valores.

Pero es también la propia historia de nuestra disciplina la que nos advierte del sesgo con el que ella misma se ha ido configurando a lo largo de las últimas décadas. Parece que sólo el mercado ha sido el sujeto digno de estudio, dejando lo jerárquico y lo valorativo abandonado fuera de los centros de atención del análisis, y llegando casi a practicar la nada científica asociación entre extramercado y extraeconómico.

El mundo jerárquico es un espacio de poder en el que la ACTIVIDAD de los individuos se regula por órdenes, de la misma forma que en el espacio de mercado esas ACTIVIDADES se regulan por los precios y en el espacio valorativo mediante persuasiones. Y no hay más que prestar un poco de atención a lo que sucede en nuestro entorno cotidiano y planetario para observar como tanto lo jerárquico como lo valorativo absorben una buena parte del tiempo de los individuos. Es más, bajo esa perspectiva, casi nada es mercado.

Al menos en nuestro Occidente, el tiempo de trabajo de la población ocupada se utiliza mayoritariamente en la realización de actividades que quedan reguladas por órdenes, bien en los puestos de trabajo de la Administración del Estado, bien en la empresa privada. Y tanto la ACTIVIDAD dentro de la Administración, civil o militar, como en el interior de la empresa privada, como ya apuntó en su momento Coase ${ }^{11}$, pertenecen al espacio jerárquico de poder.

La población no ocupada, a su vez, realiza la mayor parte de su ACTIVIDAD de acuerdo con las persuasiones que reciben, quedando así dentro del espacio valorativo. En ambos casos, ocupados y no ocupados realizan sus ACTIVIDADES mayoritariamente en espacios extramercado.

Pero tal vez resulte conveniente una pequeña reflexión sobre tales temas ${ }^{12}$.

No resultaría muy distante de la realidad admitir que la totalidad del tiempo de un individuo se reparte con arreglo a la triple división que la jerarquía, el mercado y los valores imponen a su vida. Puede emplear su tiempo en ejecutar las órdenes recibidas, en preparar objetos para el mercado, o dedicarlo a llevar a cabo las acciones a las que ha sido persuadido por otros o por él mismo.

11. Coase, R.H. (1937): “La Naturaleza de la Empresa". Recogido en Stigler, G.J. y Boulding, K.E. (1957). (Ver nota 2).

12. En lo que sigue recojo algunos párrafos del libro del que soy autor Jerarquía, Mercado, Valores: una reflexión económica sobre el poder: Alianza Editorial. Madrid, (1992). 
Llamaremos entonces “ACTIVIDAD” al empleo concreto del tiempo de los individuos regidos por órdenes, precios o persuasiones. Las ocho horas diarias de trabajo de un individuo sujeto al reglamento de una empresa, así como el tiempo dedicado a la confección familiar de bolsos de piel para venderlos en el mercado o a un intermediario, o las horas dirigidas al cuidado de un hijo, la preparación de alimentos o limpieza del hogar, o la asistencia a un acto religioso, constituyen ejemplos de tiempo consumidos en ACTIVIDADES regidas por órdenes, precios y persuasiones.

Así, si su ACTIVIDAD se rige por órdenes diremos que ese individuo pertenece a un espacio jerárquico; si lo hace por precios a uno de mercado; y si realiza su ACTIVIDAD mediante persuasiones, a un espacio valorativo.

Y de nuevo persiste el número tres, puesto que lo dicho debe ser inmediatamente matizado a un triple nivel.

En primer lugar cada individuo puede pertenecer simultáneamente a los tres órdenes de poder: bastaría con imaginarnos una simple división de las veinticuatro horas del día en tres veces ocho para considerar esa triple pertenencia.

En segundo lugar puede existir una compatibilidad desde el punto de vista del individuo para la pertenencia simultánea -en el mismo tiempo-a los tres sistemas. Alguien, por ejemplo, puede trabajar bajo órdenes en una empresa textil mientras que aprende lo necesario para aplicarlo a su taller doméstico, y pensar al mismo tiempo que ese duro trabajo contribuye a su salvación eterna o a su prestigio social o grupal.

En tercer lugar, y como ya antes apuntábamos, difícilmente podrá encontrarse una regulación de la ACTIVIDAD basada exclusivamente en órdenes, o en precios, o en persuasiones. La mezcla es lo habitual.

Y esa triple matización exige una separación tajante entre lo que hemos denominado "ACTIVIDAD" y aquello otro a lo que llamaremos "MOTIVACIÓN".

La ACTIVIDAD es la forma concreta en la que se entrega el tiempo. Tal ACTIVIDAD puede estar regida fundamentalmente por órdenes, o por precios, o por persuasiones. Pero, como también hemos dicho, difícilmente podremos encontrar una ACTIVIDAD dirigida exclusivamente por uno de los tres sistemas. Sin embargo, siempre podremos encontrar, por profunda que sea la mezcla, uno de ellos que sea indispensable para que la ACTIVIDAD se realice.

Puede que la ACTIVIDAD en una empresa concreta no se provoque exclusivamente por el cumplimiento de órdenes directas, rutinas o reglamentos, en tanto que puedan venir acompañados de premios por tareas o esfuerzos especiales, o de elementos persuatorios tales como quedar bien delante del agradable jefe o destacar dentro del grupo de trabajo. 
Resulta obvio, no obstante, que el funcionamiento de la empresa requiere indispensablemente el sistema de órdenes. Una empresa podría realizar su ACTIVIDAD productiva sin recurrir a compensaciones ni a elementos valorativos, pero nunca podría prescindir del sistema jerárquico sea cual sea la forma que adopten las órdenes que desde éste se emitan. Por ello, siempre que nos encontremos con una ACTIVIDAD regulada de forma fundamental -esto es, indispensable-por órdenes, diremos que esa ACTIVIDAD se realiza dentro de un espacio jerárquico.

El espacio de mercado, por el contrario, es aquel en que la ACTIVIDAD de los individuos queda regulada por un sistema de precios. Los precios actúan como señales que indican al individuo qué debe hacer en cada momento concreto.

Y, nuevamente, difícil será imaginarse la realización de una ACTIVIDAD de mercado en la que el sistema de precios no se vea acompañado de elementos jerárquicos y valorativos. Mientras que un individuo realiza una transacción de mercado puede verse sujeto a un impuesto jerárquico, y también relacionarse valorativamente al conversar con el resto de los participantes. El sistema de precios se revela aquí como el factor indispensable. Se podría, teóricamente al menos, prescindir de todo elemento jerárquico y valorativo, pero no podríamos prescindir de los precios. Por ello, siempre que nos encontremos con una ACTIVIDAD regulada de forma fundamental -esto es, indispensable- por el sistema de precios, diremos que esa ACTIVIDAD se realiza dentro de un espacio de mercado.

Y, por último, y tal vez con la que sea el ejemplo típico de una organización valorativa, en una familia podemos encontrar elementos valorativos, jerárquicos y de mercado. Pero si desaparece lo valorativo desaparece el mismo concepto familiar. Aquí es el retículo de valores el elemento imprescindible. Si las persuasiones desempeñan el papel fundamental como reguladoras de la ACTIVIDAD de los individuos, diremos que estos están incluidos en un espacio valorativo.

Será pues la forma fundamental -en el sentido que hemos explicado- en la que se regula la ACTIVIDAD de los individuos la que permitirá asignarlos a los espacios de poderes jerárquico, de mercado o valorativo, independientemente de su MOTIVACIÓN.

Llamaremos "MOTIVACIÓN" a la razón por la que un individuo acepta someterse a un espacio de poder. Y esa MOTIVACIÓN podrá ser jerárquica, de mercado o valorativa, independientemente de la ACTIVIDAD que realice.

Diremos que un individuo pertenece a un espacio de poder jerárquico, por ejemplo, un soldado en un ejército cuando su ACTIVIDAD se rige fundamen- 
talmente por órdenes, independientemente de su MOTIVACIÓN, que en nuestro ejemplo podría ser jerárquica -derecho a parte del botín-de mercado - a cambio de un sueldo- o valorativa -los honores y el respeto del uniformeo bien, probablemente, una mezcla adecuada de los tres componentes.

Análogamente diremos que alguien se somete al mercado cuando la regulación de su ACTIVIDAD sea fundamentalmente mediante el sistema de precios, independientemente de la MOTIVACIÓN jerárquica, de mercado o valorativa que le lleve a participar en el mercado. Por último, un individuo pertenecerá a un espacio valorativo siempre que su ACTIVIDAD quede condicionada fundamentalmente por persuasiones, independientemente del hecho de que la MOTIVACIÓN para aceptar esa sumisión pertenezca a cualquiera de los tres sistemas.

El dual del sometimiento será, lógicamente, la organización. Así hablaremos de organizaciones jerárquicas, o simplemente Jerarquía, de organizaciones de mercado, o simplemente Mercado, y de organizaciones valorativas, o simplemente Valores, cuando el procedimiento fundamental utilizado para regular la ACTIVIDAD de los individuos sometidos sea, respectivamente, las órdenes, los precios, y las persuasiones.

Denominaremos, por último, "FINALIDAD" a la ACTIVIDAD de la propia organización, esto es, al mecanismo que determina la dirección última que tomará la combinación de los tiempos de los individuos a ella sometidos.

\section{LA TEORÍA ECONÓMICA DE LA TEORÍA ECONÓMICA}

No se trata de establecer aquí lo que "producimos" como teóricos de la Economía. Creo que es algo más que los, deliciosamente descritos, "models" de Leijonhufvud. Pero sea lo que sea, algo hacemos con nuestro tiempo, y su resultado no siempre es una simple mercancía. La secuencia tópica PopperFeyerabend-Kuhn-Lakatos, sugiere que algo de sociedad extraña formamos como pretendidos científicos, y en consecuencia, tal vez, volviendo reiteradamente a Friedman, podríamos decir que la Teoría Económica de la Teoría Económica sería la ciencia que estudiaría cómo una determinada sociedad -la de los teóricos- resuelve el problema económico, entendiendo éste como la existencia de recursos escasos para la consecución de objetivos alternativos.

No deseo discutir aquí si tal pretendida ciencia lo es o no. Basta, creo, con suponer que se comporta "como si" lo fuera. Queda claro que la "determinada sociedad" somos nosotros mismos; que lo de "determinada" hace alusión a la necesaria historia de nuestro grupo de pertenencia, y que lo de "sociedad" 
recoge la idea de unas formas colectivas o conjuntas, esto es generales o habituales, de resolver el problema, y no simplemente la trayectoria concreta e individual que haya seguido uno de sus miembros.

Tenemos, por otro lado, un recurso claramente escaso: nuestro tiempo, y una clara posibilidad para utilizarlo en distintas construcciones teóricas, enfrentarnos con diversos problemas, usar distintas técnicas, y tratar de comunicar el contenido de nuestros "productos" por variados caminos.

$\mathrm{Y}$, lógicamente, nuestro sistema deberá solucionar las tres preguntas tradicionales del "¿Qué?", el "¿Cómo?” y el "¿Para Quién?" utilizando las tres formas también tradicionales: la Jerarquía, el Mercado y los Valores.

Más concretamente, entonces, deberíamos preguntarnos: ¿Cómo se regula la ACTIVIDAD de los teóricos?, ¿Cuál es su MOTIVACIÓN?, ¿Cuál es la FINALIDAD de la organización a la que pertenecen?

Creo que podría acordar, en primer lugar, que raro es el teórico de la economía cuya ACTIVIDAD fundamental quede regulada por los precios. No suele ser habitual la existencia de un mercado que remunere directamente los "productos" teóricos fruto de la ACTIVIDAD de un individuo concreto y aislado. Por supuesto que podría citarse casos singulares vinculados normalmente con algún negocio editorial, pero siempre resultarían la excepción y no la regla; y recordemos que se trata, como de la propia definición se desprendía, de observar las formas colectivas, generales, con las que se resuelve el problema económico.

Y por lo general, aunque el producto de la ACTIVIDAD suele tener el carácter de creación individual, los teóricos lo realizan dentro de una organización, particularmente Universidades e Institutos de Investigación. Y como dentro de esas organizaciones no suele regir el sistema de precios, serán las reglamentaciones jerárquicas y las persuasiones valorativas los reguladores básicos de la ACTIVIDAD teórica.

La mezcla órdenes -persuasiones varía normalmente con la antigüedad en la organización. Al inicio el individuo recibe una combinación fuertemente cargada de elemento jerárquico, pero según va pasando el tiempo, y ascendiendo por la escala jerárquica y valorativa, su ACTIVIDAD tiende cada vez más a quedar regulada por elementos valorativos. Del "esto es lo que hay que saber, estudiar, conocer, aprender y realizar" se pasa poco a poco a "esto es lo que es mejor saber, estudiar, conocer, aprender y realizar". La sumisión jerárquica deja paso paulatinamente a la aceptación valorativa.

Como en cualquier otra organización poco podemos decir sobre la MOTIVACIÓN que impulsa a los individuos a incorporarse al espacio jerárquico y valorativo en el que realizarán su ACTIVIDAD. Unicamente podemos hablar 
de lo que sus preferencias parecen revelar.

La participación en la organización está generalmente remunerada monetariamente, luego la MOTIVACIÓN mercado existe. También se sabe que, con el paso del tiempo, se puede alcanzar cierta posición en la organización que permita emitir órdenes, con lo que tampoco es descartable una MOTIVACIÓN jerárquica. Sin embargo, tanto la baja remuneración como la escasa capacidad jerárquica que puede alcanzarse en estas instituciones, -siempre comparativamente hablando, claro está- junto con la importancia ya advertida de las persuasiones como reguladoras de la ACTIVIDAD, hacen pensar que probablemente sea el elemento valorativo el componente fundamental de la MOTIVACIÓN de tales teóricos.

Y en cuanto a la FINALIDAD, esto es a la ACTIVIDAD de la organización, nuevamente nos encontramos con la mezcla habitual de regulación por órdenes, precios y persuasiones valorativas. La regulación jerárquica se aprecia en cosas tales como homologación de planes de estudios, reglamentos mínimos de funcionamiento, etc. El elemento de mercado aparece con la aportación de fondos ajenos para el estudio de temas concretos o el fomento de determinadas líneas de investigación. Pero de nuevo, quizá sea lo valorativo el gran determinante en última instancia de la FINALIDAD.

\section{El Mrto deL MerCAdo}

Esa mezcla de órdenes, precios y persuasiones nos impulsó, digamos que desde Smith, a pensar lo que pensamos, a escribir lo que escribimos y a decir lo que decimos. Y la verdad es que con algunas, muy pocas excepciones, nos centramos en el mercado; o bien, para ser más precisos, en el funcionamiento del sistema de precios.

La fascinación del mercado es común a todas las escuelas. Desde todos los clásicos, incluyendo de forma destacada a Marx, hasta nuestros días. Y probablemente, con un mínimo esfuerzo, esa esbozada aquí Teoría Económica de la Teoría Económica podría explicar el por qué. Pero, en estas páginas, nos basta con la constatación del hecho y con la simple insinuación de sus razones.

Y la fascinación a la que nos referíamos ha dado sus resultados. A estas alturas del desarrollo de la Teoría podemos atrevernos a decir que sabemos todo lo que economicistamente se puede saber del sistema de precios.

Y digo "economicistamente" para resaltar la situación presente en la que, utilizando los viejos y queridos conceptos heredados de la matemática del XVIII, la aportación marginal derivada del esfuerzo por conseguir nuevos 
resultados es mínima en comparación con lo que puede lograrse con ese mismo esfuerzo en cualquiera de los ámbitos de estudio extramercado.

Tal hipótesis, no significa ni mucho menos que no se siga produciendo una inmensa cantidad de literatura que trata, y de hecho lo consigue, de hacer una nueva aportación a tan establecido tronco de doctrina. Y tampoco se deduce de ella que tal comportamiento sea irracional o extraeconómico. Nuevamente, serán los ámbitos jerárquicos y valorativos los responsables de una ACTIVIDAD que no podría explicarse exclusivamente por la aplicación economicista de unos criterios marginales diseñados para el estudio de los mercados.

Sobre el mercado se sabe todo lo que, prácticamente, hay que saber; y si se continúa con su estudio será por razones, honestas y racionales, económicas si se quiere, pero ajenas a la construcción eficiente de un conocimiento científico.

Conocemos precisamente las condiciones requeridas para la "competencia perfecta" y para el establecimiento de un equilibrio general competitivo. Y sabemos asimismo las implicaciones que ello tiene sobre el bienestar.

Todavía no está claro si tales modelos puros se construyeron por sus respectivos creadores pensando que de alguna forma reflejaban en ellos la "realidad" cotidiana o simplemente fueron resultado de un deseo de establecer los requisitos exigidos para un funcionamiento correcto y completo de todo el sistema de precios.

Sea como fuere, lo que si está claro es que tal "realidad" ofrece, se mire como se mire y en cualquier momento de la historia, unas características que permiten asegurar, debido precisamente al conocimiento riguroso que teóricamente se tiene del funcionamiento del mercado, que tal sistema a la hora de resolver el llamado problema económico, ni es el único, ni es el óptimo.

Podríamos, en este momento, exponer una serie de características de esa llamada realidad que invalidarían necesariamente esas conclusiones de bienestar que del funcionamiento de los mercados puros suele deducirse. Bastaría, por ejemplo, con hacer referencia a las situaciones no competitivas, a la no existencia de mercados de futuros para cada uno de los posibles bienes en las distintas situaciones, a la imposibilidad de establecer un retículo completo de derechos de propiedad, a la no correspondencia tecnológica con las funciones de producción homogéneas y de grado uno, a la existencia de funciones de utilidad interdependientes, etc, etc.

Bastaría con ello, digo, para, como bien sabemos, anular las conclusiones de bienestar asociadas al funcionamiento "perfecto" de los mercados. Pero aquí quiero resaltar tres características suficientemente establecidas teóricamente, que hacen que el mercado sea inútil para resolver tres problemas que pueden considerarse en nuestro hoy y ahora real como de suma importancia. 
1.- El mercado no revela, ni consecuentemente resuelve, las necesidades de los individuos. Sólo lo hace con aquellas necesidades que puedan ser respaldadas con "votos monetarios".

2.- Nada puede hacerse con el mercado respecto a los denominados bienes, o males, públicos.

3.- En presencia de tiempo histórico, aún en mercados atomísticos, puede existir exceso de oferta en uno de los mercados con equilibrio en el resto de ellos.

Dicho de otra forma, aunque todas las imperfecciones se eliminasen, aunque los derechos de propiedad se establecieran de forma extensa y totalizadora, aunque existieran mercados para todos los productos imaginables en todas las circunstancias posibles, aunque fuéramos individuos aislados exentos de altruismo y de envidia. Aunque todo eso ocurra, si el pasado está dado y no se puede cambiar y el futuro es incierto y no se puede conocer, sabemos con certeza que el mercado no puede resolver ni la pobreza, ni los problemas colectivos, ni el desempleo. Eso sí que lo sabemos.

Es más, ya que posiblemente con el tercero de los puntos podría entrarse en algún tipo de polémica entre los propios teóricos podemos fácilmente eliminarlo para buscar el máximo consenso. Así, lo que si podemos decir con total convicción sería que, incluso en el mundo utópico donde rigiese la más perfecta de todas las competencias, y con un tiempo lógico que permitiese, por ejemplo, tener hijos y luego decidir si se tienen. Aún en ese mundo sólo se revelarían las necesidades respaldadas con votos monetarios, y nada podría hacer el mercado con los bienes públicos. En ese mundo "perfecto" algo habría que hacer extramercado para reparar la muerte de niños hambrientos y eliminar el agujero de ozono. El mercado no sirve para resolver esos problemas. Eso, nuevamente, si que lo sabemos.

Por todo ello, si una sociedad, por muy perfectamente competitiva que sea, estima que la pobreza es un problema y se percata de que tiene bienes y males que trascienden lo individual y entran en la esfera de lo público, sabrá que no puede recurrir al mercado para enfrentarse con tales temas. Si además advierte que la moviola del tiempo sólo la pueden utilizar ciertos brujos, consciente de su tiempo histórico, se dará cuenta de que el mercado tampoco puede resolver problemas como el del desempleo. Y si, adicionalmente, comienza a observar que esa tal "perfección" no existe en tal o cual tema, deberá pensar consecuentemente que tampoco puede recurrirse al mercado para esto o para lo otro. Y así, probablemente, llegará al estadio en el que toda sociedad, actual o pasada, se establece. Con una mezcla necesaria de Jerarquía, Mercado y Valores. 


\section{ODRES NUEVOS PARA EL NUEVO VINO}

Coexistimos en la profesión muchas clases de teóricos. Y mal que pueda pesarle a alguien, hay espacio para todos. Dentro de todos ellos, algunos pensamos que la Teoría Económica que estudiamos y hacemos debe servir para la interpretación, la explicación y el análisis del cambio social, sintonizando así con lo que estimamos que era la intención de los clásicos y de toda una serie de economistas posteriores con los que nos mantenemos, creemos, unidos en la intención.

Adicionalmente, y respetando el trabajo duro y honrado de tantos otros dedicados a tantos otros temas, pensamos que ese Cambio Social no tiene una neutralidad valorativa. Los problemas que se nos plantean no son sólo explicativos, sino éticos.

La pobreza y la miseria, el hambre y la desesperación no son para nosotros simples aspectos de una evolución social. También, y algunas veces sobre todo, nos duelen. La marginación, el racismo, el individualismo extremo, la tortura y la degradación, el miedo... no son sólo facetas observables, y susceptibles de ser explicadas, de una realidad cambiante. También, y algunas veces sobre todo, nos duelen.

Las matanzas y las guerras, las máquinas militares en toda su compleja extensión masacrando miles de individuos... no sólo son para nosotros fenómenos tecnológicos y sociales de lucha entre las tribus desde los primeros peldaños de nuestra historia hasta nuestros días. También, y algunas veces sobre todo, nos duelen.

El caos de las ciudades, la polución y el ruido, la desaparición continua y creciente de especies animales y vegetales que forman con nosotros la vida de nuestro mundo. Los desastres ecológicos cotidianos, el envenenamiento y la contaminación creciente del medio... no sólo son para nosotros fenómenos observables en la interrelación planeta-tiempo. También, y algunas veces sobre todo, nos duelen.

Puede ser que, para algunos, estas afirmaciones nos descalifiquen como científicos. Allá ellos. Ni vivimos para conquistar ese calificativo, ni ignoramos tampoco el ridículo científico en que incurrirían, en estos tiempos, los que osen emitir bulas de cientificidad.

Lo que si ocurre es que, científicos o no -que ya decidirá la historia futura lo que quede de nuestro presente- teóricos si que somos. Lo suficiente como para saber con completa certeza que el mercado no puede utilizarse para resolver ni uno sólo de los problemas que acabamos de mencionar. Ni uno sólo.

Y además, también como teóricos que somos, formulamos una hipótesis que 
el tiempo -aunque hasta ahora no lo ha hecho- posiblemente se encargará de falsar: la extensión política del mercado agravará tales problemas. El mercado no es neutral.

Pero en la línea anterior de esta misma página se hace referencia a "tales problemas". Y los problemas sólo existen si se definen como tales. A algunos, la pobreza y lo colectivo nos parecen simplemente importantes en nuestro hoy y ahora. Mi confianza, y ahora personalizo, es que terminarán siendo importantes no ética, sino jerárquicamente. Y trato de explicar, personalizando aún más, el por qué.

Un problema sólo es tal si así se define. Es decir, que lo será más en cuanto más definido esté. Nos movemos, los teóricos, por aquella mezcla de jerarquíamercado - valores a la que hace unas páginas nos referíamos. Y los valores pesan sobre eso de la pobréza, que existe, y esos problemas colectivos, que también existen. Pero también pesa la jerarquía: la definición concreta de los temas a estudiar, por así decirlo.

Y la jerarquía pesa mucho. Tanto que, probablemente, $-\mathrm{y}$ esto es otra hipótesis- hasta que la Jerarquía no tome y configure como suyo el asunto de la pobreza y los temas de bienes y males públicos, poca será la movilización de la gran masa de los teóricos hacia esos aspectos de la llamada realidad.

Y quizá la pobreza comience a preocupar de nuevo a la Jerarquía. Y quizá lo público, lo planetario comience a ser, por las razones que sean, un problema para el Gran Orden.

Entonces tocarán los timbales sordos, los ocultos. Y mientras que en la Prensa se seguirá haciendo publicidad del mercado como si fuera un remedio para los sabañones, los grandes centros de investigación, no propagandísticos, sino reales, tendrán necesariamente que hacer frente al menos a estos dos grandes temas.

La investigación publicitaria seguirá creando eslóganes de mercado mientras el mundo se debate en medio de sangre y hambre. Pero la investigación teórica, no sólo la motivada éticamente sino también la otra, deberá presentar resultados si quiere sobrevivir como algo más que una simple productora de diversión.

Esos temas creo que configurarán nuestro descastado y bravío vino nuevo al que tendremos necesariamente que trasegar.

Y para ello, por algo sabemos lo que sabemos, no nos bastará la sabiduría acumulada sobre el mercado. Esa sabiduría nos sirve precisamente para saber que no sirve.

No nos valdrá para esta tarea el viejo pensamiento neoclásico aunque se arrope con la etiqueta de Nuevo. Y de poco más que de entrenamiento en el rigor 
serán de utilidad las antiguas recetas marginalistas y las formulaciones matemáticas al uso.

Creo que son necesarios odres nuevos para contener este nuevo vino. Quizá, sólo quizá, sea necesaria una reformulación de la Economía como reflexión sobre el poder. ¿Qué se hace con él? ¿Cómo se lleva a la práctica?, Quién lo disfruta?.

La nueva Economía Política podría -otra vez quizá- tratar de entender dos cosas muy simples: “¿Cómo se genera el poder?” y “¿Cómo se distribuye?”. Producción y Distribución, viejos conceptos queridos por todos los que de una u otra forma, y por una u otra razón dejamos en tales reflexiones nuestro tiempo.

Todo esto, por supuesto, es un deseo. Pero también una invitación. Además, eso espero, es casi una necesidad. 